\title{
Genome-wide identification of tissue- specific long non-coding RNA in three farm animal species
}

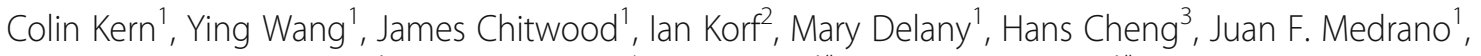
Alison L. Van Eenennaam ${ }^{1}$, Catherine Ernst ${ }^{4}$, Pablo Ross ${ }^{1 *}$ and Huaijun Zhou ${ }^{1 *}$

\begin{abstract}
Background: Numerous long non-coding RNAs (IncRNAs) have been identified and their roles in gene regulation in humans, mice, and other model organisms studied; however, far less research has been focused on IncRNAs in farm animal species. While previous studies in chickens, cattle, and pigs identified IncRNAs in specific developmental stages or differentially expressed under specific conditions in a limited number of tissues, more comprehensive identification of IncRNAs in these species is needed. The goal of the FAANG Consortium (Functional Annotation of Animal Genomes) is to functionally annotate animal genomes, including the annotation of IncRNAs. As one of the FAANG pilot projects, IncRNAs were identified across eight tissues in two adult male biological replicates from chickens, cattle, and pigs.

Results: Comprehensive IncRNA annotations for the chicken, cattle, and pig genomes were generated by utilizing RNA-seq from eight tissue types from two biological replicates per species at the adult developmental stage. A total of 9393 IncRNAs in chickens, 7235 IncRNAs in cattle, and 14,429 IncRNAs in pigs were identified. Including novel isoforms and IncRNAs from novel loci, 5288 novel IncRNAs were identified in chickens, 3732 in cattle, and 4870 in pigs. These transcripts match previously known patterns of IncRNAs, such as generally lower expression levels than mRNAs and higher tissue specificity. An analysis of IncRNA conservation across species identified a set of conserved IncRNAs with potential functions associated with chromatin structure and gene regulation. Tissue-specific IncRNAs were identified. Genes proximal to tissue-specific IncRNAs were enriched for GO terms associated with the tissue of origin, such as leukocyte activation in spleen.
\end{abstract}

Conclusions: LncRNAs were identified in three important farm animal species using eight tissues from adult individuals. About half of the identified IncRNAs were not previously reported in the NCBI annotations for these species. While IncRNAs are less conserved than protein-coding genes, a set of positionally conserved IncRNAs were identified among chickens, cattle, and pigs with potential functions related to chromatin structure and gene regulation. Tissue-specific IncRNAs have potential regulatory functions on genes enriched for tissue-specific GO terms. Future work will include epigenetic data from ChIP-seq experiments to further refine these annotations.

Keywords: Long non-coding RNAs, Gene regulation, Epigenetics

\footnotetext{
*Correspondence: pross@ucdavis.edu; hzhou@ucdavis.edu

'Department of Animal Science, University of California, Davis, Davis, CA, USA

Full list of author information is available at the end of the article
}

C The Author(s). 2018 Open Access This article is distributed under the terms of the Creative Commons Attribution 4.0 International License (http://creativecommons.org/licenses/by/4.0/), which permits unrestricted use, distribution, and reproduction in any medium, provided you give appropriate credit to the original author(s) and the source, provide a link to the Creative Commons license, and indicate if changes were made. The Creative Commons Public Domain Dedication waiver (http://creativecommons.org/publicdomain/zero/1.0/) applies to the data made available in this article, unless otherwise stated. 


\section{Background}

Since the invention of genome sequencing technology, the focus of genomics has been to identify the genes present in an organism and understand their link to traits, or phenotypes, that the organism exhibits. As more is learned about genetics and the key role gene regulation plays in phenotypic expression, it is becoming clear that a complete understanding of the genome-to-phenome relationship will require a more comprehensive annotation of the genome than just protein-coding genes. RNA-seq data has revealed that while less than $5 \%$ of the human genome consists of protein coding sequences, most of the genome is transcribed [1-3]. Furthermore, comparative genome studies have shown evolutionary conservation in intergenic regions of the genome, indicating positive selection pressure and implying that these conserved regions have important functions [4-7].

One class of important regulatory elements that has recently been gaining attention is long non-coding RNAs (lncRNAs). These transcripts are distinct from miRNAs, snoRNAs, and others in that they are defined as greater than 200 bases in length and share some characteristics of mRNA, such as polyadenylation. LncRNAs were originally thought to not contain open reading frames (ORFs), however some have been found with short ORFs that may be translated, though the function of these is still a topic of debate $[8,9]$. Some lncRNAs have been shown to have functions in regulating gene expression. $X I S T$, for example, is a lncRNA that acts as one of the major components of the X-inactivation process in placental mammals [10]. HOTAIR is another lncRNA found on human chromosome 12. High expression of this lncRNA in breast cancer tumors is a significant predictor of metastasis [11]. HOTAIR is particularly notable as it was the first RNA discovered that is transcribed from one chromosome and regulates transcription of a gene on a different chromosome. Another IncRNA, Malat1, has been studied in mice and shown to affect the expression of neighboring genes on the same chromosome [12]. Long non-coding RNAs can therefore regulate genes in both cis and trans, demonstrating the importance of studying these molecules.

Many studies have identified genome-wide lncRNAs in model organisms such as human [13-18], mouse [18-22], zebrafish [23, 24], frog [25], fruit fly [26, 27], nematode [28], and Arabidopsis [29]. Some IncRNA identification efforts have focused on maize [30] and one of the primary malaria-causing parasite species, Plasmodium falciparum [31]. For farm animals, work has begun more recently to identify lncRNAs in chickens [32-37], cattle [38-43], pigs [33, 44-48], sheep [49-52], goats [53-56], and horses [57]. A recent review of lncRNA in livestock species provides a comprehensive overview of the current progress in the field [58]. Many of the lncRNA studies in livestock were performed using samples from varied developmental stages or using only one or two tissues while comparing between a control and experimental conditions. The chicken, cattle, and pig genomes are still lacking a comprehensive genome-wide catalog of lncRNAs in multiple tissues from adult animals.

The efforts of the ENCODE projects in creating comprehensive functional annotations of the human and mouse genomes have become a model for the Functional Annotation of Animal Genomes (FAANG) Consortium [59], whose goal is to functionally annotate all farm animal genomes. As one of the FAANG pilot projects, 48 tissue samples were collected from eight tissues across two biological replicates from chickens, cattle, and pigs. Adult male animals were used as they represent a transcriptionally stable state, avoiding the relatively more dynamic gene expression associated with development, growth, and the female reproductive cycle in certain tissues. Biological replicate animals were chosen to minimize biological diversity in each species. A highly inbred line was used for the chicken, the pigs sampled were littermates, and both cattle replicates had the same sire and were from a cattle line closely related to the cattle sequenced to construct the reference genome. The tissues were selected to include those that have a large number of associated quantitative phenotypic traits, focusing on traits relevant to the food production industry such as growth, health, feed efficiency, and disease resistance. The set of eight tissues used consisted of skeletal muscle, adipose, liver, lung, spleen, cerebellum, cortex, and hypothalamus.

As part of a FAANG pilot project, 48 stranded RNA-seq libraries were generated to identify lncRNAs in eight tissues from two biological replicates across the genomes of chicken, cattle, and pig. Using data from the same eight tissues in each species enabled the identification of tissue-specific lncRNAs, as well as those that appear to be generally expressed across the eight tissues examined. Finally, a comparative analysis of lncRNAs with shared expression between the three species was conducted to study evolutionary conservation of lncRNAs.

\section{Results \\ Identification of IncRNAs}

Since lncRNAs are generally expressed at low levels [17] and can be hard to separate from noise in the data, the use of two biological replicates helped to verify the reproducibility of the results. Filtered and aligned RNA-seq reads (Table 1) for each of the eight tissues surpassed 100 million reads, a recommended threshold for identifying novel isoforms or transcripts that are expressed at low levels [60]. Table 2 and Table 3 show the number of genes and transcripts assembled for each RNA-seq library individually, which were then merged into a common transcriptome across all tissues. The number of transcripts in 
Table 1 Total number of aligned and filtered RNA-seq reads per tissue

\begin{tabular}{llll}
\hline & Chicken & Cattle & Pig \\
\hline Adipose & $198,929,564$ & $156,656,620$ & $119,721,691$ \\
Cerebellum & $242,807,223$ & $246,658,282$ & $152,762,359$ \\
Cortex & $236,147,593$ & $119,721,576$ & $126,240,107$ \\
Hypothalamus & $244,215,661$ & $142,709,163$ & $132,786,659$ \\
Liver & $244,674,805$ & $119,617,850$ & $104,210,750$ \\
Lung & $205,055,604$ & $138,746,254$ & $198,053,139$ \\
Muscle & $238,435,618$ & $140,106,635$ & $155,724,909$ \\
Spleen & $201,084,991$ & $150,804,156$ & $125,682,422$ \\
\hline
\end{tabular}

the merged transcriptome that were assigned each of the Cufflinks class codes, which indicate the relationship to previously annotated transcripts, are shown in Table 4. LncRNAs were identified by comparing them with known protein-coding genes in the NCBI annotations and with known proteins across any species in the Pfam [61] and Swiss-Prot [62] databases (Fig. 1a). A total of 31,057 lncRNAs were identified across chicken, cattle, and pig (Fig. 1b). The sequences are available in Additional files 1, 2 and 3 and their genomic locations and structures in Additional files 4, 5 and 6 Each lncRNA was placed into one of three categories based on the NCBI annotation for that species: previously annotated lncRNAs, novel isoforms of annotated lncRNAs, or transcripts from novel IncRNA loci (Fig. 1c, Table 5). On average, half of lncRNAs were previously annotated; however, a larger percentage of the lncRNAs from pig were previously annotated. In all three species, more novel lncRNAs are from novel loci rather than new isoforms of previously annotated lncRNAs. Including both novel isoforms and lncRNAs from novel loci, 5288 novel lncRNAs were identified in chickens, 3732 in cattle, and 4870 in pigs. LncRNAs were also compared to the NONCODEv5 database using sequence similarity [63]. Only $7.77 \%$ of predicted chicken lncRNAs and $5.57 \%$ of cattle lncRNAs had sequences similar to those in the NONCODE database, defined as having at least $50 \%$ sequence identity and the alignment covering at least $50 \%$ of the predicted lncRNA. In pigs, $37.59 \%$ of predicted lncRNAs were similar to those in the NONCODE database. These results are summarized in Table 6, and the individual lncRNAs with their matching NONCODE IDs are in Additional file 7.

While a coding potential score was not used for indentification of lncRNAs for this study, scores were calculated by FEELnc [64] that can be used as a confidence metric for further filtering of candidates. Using the default cutoff for calling a transcript coding or non-coding by FEELnc, 996 chicken lncRNAs, 475 pig lncRNAs, and 1326 cattle lncRNAs had scores predicting them as coding. This corresponded to $11.9,3.4$, and $22.4 \%$ of candidate IncRNAs respectively.

The number of exons, transcripts, and length of lncRNAs and mRNAs are shown in Fig. 1d-f. In all three species, the majority of mRNAs contain at least 5 exons, while most lncRNAs contain only 2 or 3 exons (see Fig. 1e), which is consistent with findings from the human ENCODE project [65]. Figure 1d shows the distribution of the lengths of lncRNAs and mRNAs, which were similar within each species. However, there were differences between species that are present in both lncRNAs and mRNAs. In pigs, about $50 \%$ of both types of RNA were in the 200-999 bp range, whereas only about $25 \%$ were in this range in chickens, and cattle were in-between. A general trend was observed where chicken transcripts of both types were generally longer than cattle and pig, while pig was the shortest.

\section{Potential regulatory targets of IncRNAs}

To analyze potential regulatory function, each lncRNA was paired with the nearest protein-coding gene as a potential regulator of that gene. If no gene was within $50 \mathrm{~kb}$ upstream or downstream of a lncRNA (in other words, the distance between the transcribed regions), that lncRNA was not included in this analysis. Excluded lncRNAs represented $12.9 \%$ of lncRNAs in chickens, $16.8 \%$ of lncRNAs in cattle, and $21.5 \%$ of lncRNAs in pigs. Over $90 \%$ of all three genomes are distally intergenic enough to exclude any lncRNA by the above

Table 2 The number of genes assembled from each RNA-seq library

\begin{tabular}{|c|c|c|c|c|c|c|}
\hline & Chicken A & Chicken B & Cattle A & Cattle B & Pig A & Pig B \\
\hline Adipose & 25,837 & 27,020 & 50,396 & 51,271 & 49,322 & 47,401 \\
\hline Cerebellum & 33,830 & 33,729 & 70,001 & 81,189 & 60,174 & 66,127 \\
\hline Cortex & 35,110 & 35,984 & 46,410 & 52,946 & 50,951 & 51,532 \\
\hline Hypothalamus & 33,437 & 34,457 & 53,784 & 54,949 & 53,811 & 46,592 \\
\hline Liver & 25,127 & 27,235 & 45,275 & 47,518 & 43,793 & 44,592 \\
\hline Lung & 30,680 & 29,747 & 50,051 & 59,447 & 66,299 & 61,041 \\
\hline Muscle & 23,414 & 23,417 & 39,334 & 38,960 & 43,307 & 42,422 \\
\hline Spleen & 30,927 & 31,752 & 56,125 & 62,107 & 61,337 & 57,744 \\
\hline
\end{tabular}


Table 3 The number of transcripts assembled from each RNA-seq library

\begin{tabular}{|c|c|c|c|c|c|c|}
\hline & Chicken A & Chicken B & Cattle A & Cattle B & Pig A & Pig B \\
\hline Adipose & 66,252 & 67,811 & 96,844 & 98,317 & 90,838 & 88,337 \\
\hline Cerebellum & 76,797 & 76,515 & 119,305 & 131,204 & 104,161 & 110,994 \\
\hline Cortex & 78,157 & 79,363 & 92,521 & 100,484 & 93,695 & 94,132 \\
\hline Hypothalamus & 76,096 & 77,811 & 101,482 & 103,398 & 97,113 & 88,079 \\
\hline Liver & 64,847 & 68,013 & 90,252 & 93,361 & 80,706 & 80,826 \\
\hline Lung & 72,857 & 71,558 & 97,876 & 108,481 & 111,665 & 105,423 \\
\hline Muscle & 61,921 & 61,825 & 82,076 & 81,887 & 82,664 & 81,214 \\
\hline Spleen & 73,368 & 74,021 & 103,069 & 110,812 & 105,930 & 101,208 \\
\hline
\end{tabular}

criteria, yet not even a quarter of lncRNAs were found in these regions. This reinforces the potential regulatory roles that lncRNAs may have on genes. The remaining lncRNAs were then labeled as intergenic if they did not overlap the annotated gene region, exonic if they overlapped an exon by at least $1 \mathrm{bp}$, and intronic if they overlapped only introns (Fig. 2a). The exonic and intronic lncRNAs were then categorized based on whether they were on the same strand (sense) or opposite strand (antisense) of the protein-coding gene (Fig. 2b), while the intergenic lncRNAs were categorized by strand and by whether they were upstream or downstream based on transcriptional direction of the coding gene (Fig. 2c). Table 7 shows in detail the number of lncRNAs in each of these groups. Many exon-overlapping lncRNAs overlapped only small portions of exons. Other lncRNA exons overlapped a full protein-coding exon, but contain novel exons that do not appear to be part of an annotated gene. Regardless of the nature of the overlap, the resulting lncRNA does not have any similarity to known protein-coding transcripts or exhibit similarity to any known protein domain, and therefore may be a noncoding isoform of the gene.

In all three species, about $25 \%$ of the lncRNAs that were included in this analysis overlap the genic region, with the other $75 \%$ divided evenly between upstream or downstream location relative to the protein-coding gene. While the lncRNAs within the downstream region of genes did not appear to have any strand correlation with

Table 4 The number of each Cufflinks "class code" in the transcriptome merged from all tissues

\begin{tabular}{lllllll}
\hline & $=$ & $j$ & $u$ & $x$ & 0 & $s$ \\
\hline Chicken & 49,456 & 40,620 & 21,034 & 3205 & 802 & 0 \\
Pig & 54,311 & 41,237 & 35,046 & 4306 & 925 & 7 \\
Cattle & 64,413 & 45,759 & 30,504 & 3736 & 1071 & 0 \\
\hline
\end{tabular}

"=" is a complete match of an existing transcript in the NCBI annotation. " $\mathrm{j}$ " is a potential novel isoform of an existing transcript. " $u$ " is an unknown intergenic transcript. " $x$ " is an exonic overlap on the opposite strand. "o" is an overlap with annotated exons, but is not classed as " $\mathrm{j}$ " because no splice sites match. " $\mathrm{s}$ " is an intronic overlap on the opposite strand. See http://cole-trapnell-lab.github.io/ cufflinks/cuffcompare/ for more details the gene (they were equally sense or antisense), there was a higher prevalence of antisense lncRNAs within the upstream region of genes in all three species. The Spearman correlation of the expression of the lncRNAs with their nearest genes was used to provide evidence for potential cis-regulatory function. To compare this correlation between groups and species, the average correlation was calculated for each species, then the difference was calculated from this average for each group of lncRNAs based on their positional relationship with the nearby gene, e.g. antisense upstream (Fig. 2d), and also for each tissue (Fig. 2e). A higher correlation between the expression of upstream antisense lncRNA-gene pairs was observed across all three species, supporting the potential co-regulation of these transcripts. The correlation in expression of intergenic lncRNA gene pairs was generally higher in cattle compared to chicken and pig, however in chicken the correlation was not affected by the distance of the lncRNA from the gene, while in cattle and pig shorter distances are associated with higher correlation (Fig. 2f). The lncRNA-gene pairs and their positional relationships are available as Additional files 8, 9 and 10, and the expression for every lncRNA in each sample is shown in Additional files 11, 12 and 13.

\section{Tissue-specific IncRNAs}

Tissue-specific lncRNAs were identified using a Tissue Specific Index (see Methods). Fewer tissue-specific lncRNAs were seen in brain and adipose across the three species (Fig. 3a). As lncRNAs are known to be expressed at lower levels than mRNAs [17], any cutoff would be arbitrary, therefore lncRNAs that were expressed at any non-zero level were included. The percentage of lncRNAs expressed at or above a sliding cutoff was graphed, and in all three species lncRNAs specific to liver and muscle stood out as being expressed at higher levels than other tissues (Fig. 3b-d). The Tissue Specific Index calculated for each lncRNA is shown in Additional files 14, 15 and 16. The same analysis was repeated, but instead by calculating tissue-specificity using the expression of lncRNA loci rather than the expression of individual transcripts. In other 


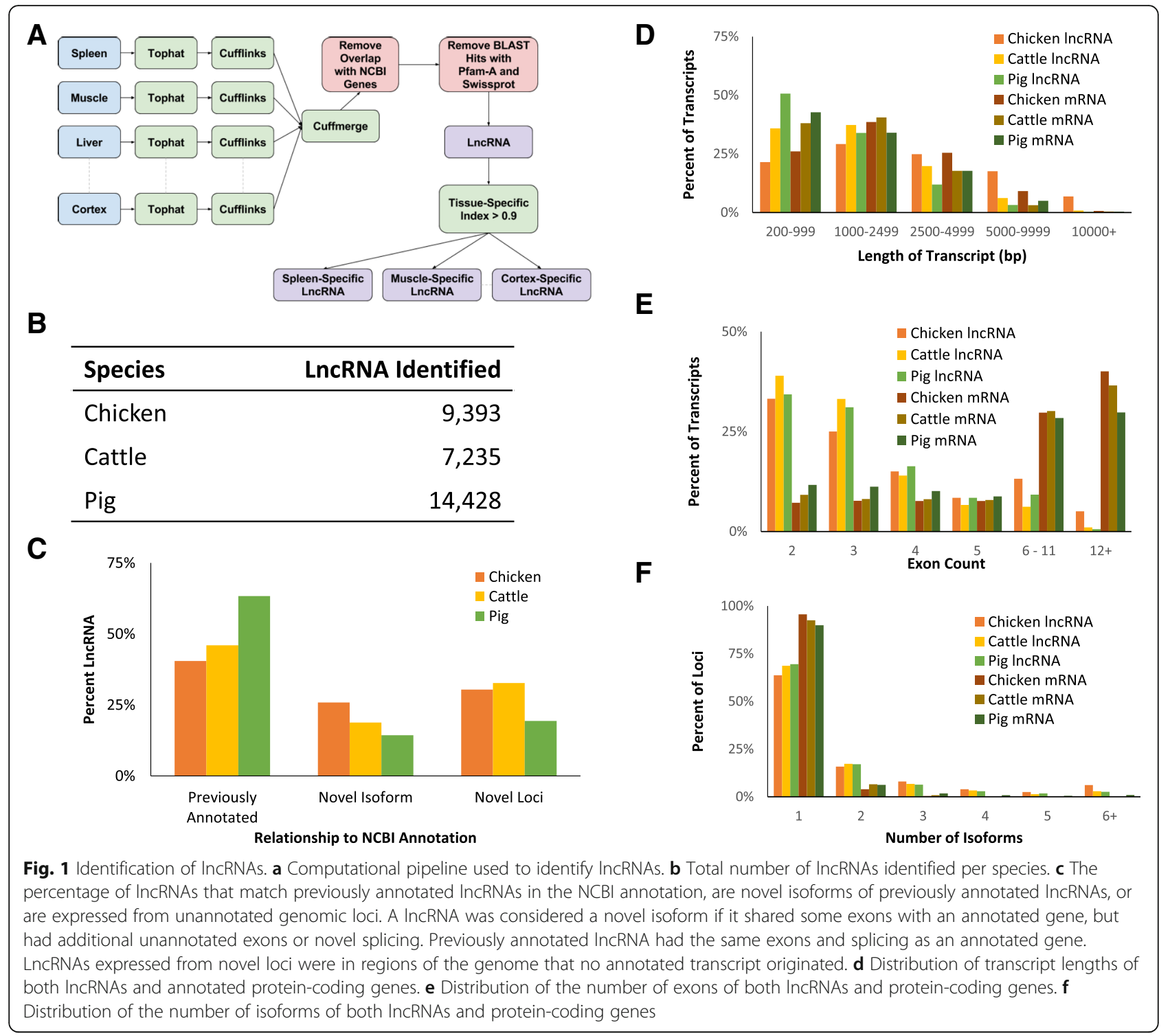

words, the expression of multiple transcripts originating from the same loci would have been measured by a single expression value. The results mirrored the trends of the transcript-level analysis and are not presented in detail.

The gene ontology (GO) terms enriched in the set of genes associated with nearby tissue-specific lncRNAs were analyzed to understand the potential regulatory function of these IncRNAs (Additional files 17, 18 and 19). The

Table $\mathbf{5}$ The number of IncRNA transcripts and loci from NCBI annotations and this study

\begin{tabular}{llllll}
\hline & Chicken & Cattle & Pig & Human & Mouse \\
\hline NCBI Transcripts & 6072 & 6187 & 14,503 & 27,986 & 21,705 \\
Novel Transcripts & 9393 & 7235 & 14,429 & - & - \\
NCBI Loci & 4167 & 4601 & 10,388 & 15,765 & 11,957 \\
Novel Loci & 4654 & 4325 & 8772 & - & - \\
\hline
\end{tabular}

tissue-specific index was calculated for these sets of associated protein-coding genes, and the percentage found to be tissue-specific is shown in Fig. 3e. On average across all species and tissues, only $17 \%$ of these genes were tissue-specific, with a maximum of $27 \%$ in cattle liver (Fig. 3e). Only two tissues had GO terms that were enriched across all three species. In cerebellum, nervous system development, generation of neurons, positive

Table 6 LncRNA comparison with the NONCODEv5 database based on sequence similarity

\begin{tabular}{llll}
\hline & Novel LncRNA & NONCODE & Overlap \\
\hline Chicken & 9393 & 12,850 & 730 \\
Pig & 14,429 & 29,585 & 5424 \\
Cattle & 7235 & 23,515 & 403 \\
\hline
\end{tabular}




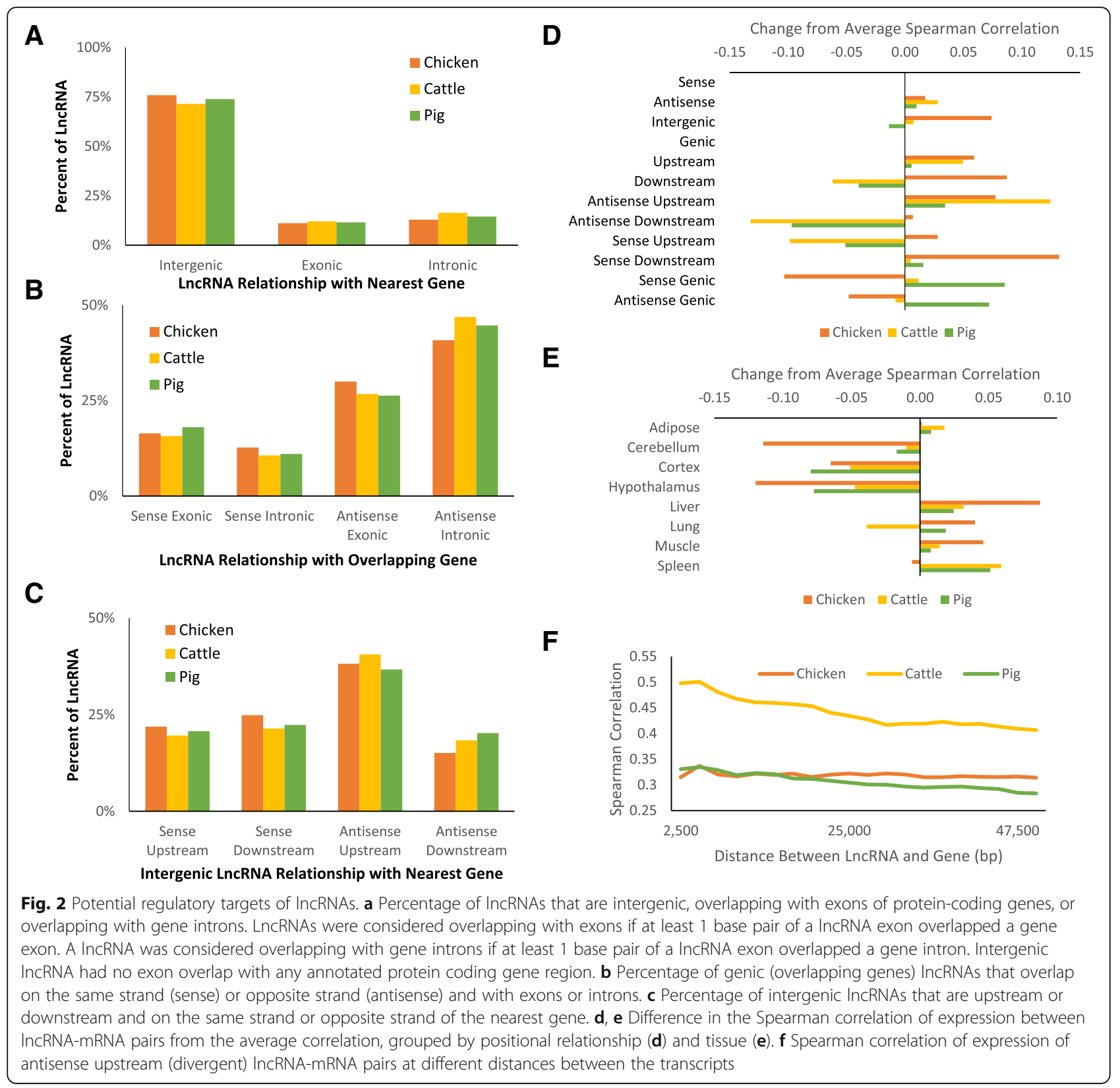

regulation of developmental process, regulation of cell differentiation, and regulation of multicellular organismal development were enriched in chicken, cattle, and pig. In cortex, nervous system development was enriched in all three species. While no other GO terms were enriched across all three species in the same tissue, related GO terms were enriched across species in some tissues, or GO terms were shared between two species. In adipose, skeletal system development was enriched in both cattle and chickens. GO terms related to the skeletal system did not appear in adipose from pigs. In addition to the GO terms shared across all species previously reported, some brain tissues contained GO terms specific to individual brain regions. Regulation of circadian rhythm was enriched by lncRNAs specific to the hypothalamus in chickens, and spinal cord development was enriched by lncRNAs specific to the cerebellum in cattle. GO terms associated with vasculature were enriched in the cerebellum and hypothalamus chicken: circulatory system development in hypothalamus, blood vessel morphogenesis in cerebellum. In liver, many metabolic process related GO terms were enriched for cattle and pig such as monocarboxylic acid metabolic process in cattle and alcohol metabolic process in pig; however, these were absent in chickens. No GO terms were significantly enriched for lung in chickens, but in cattle and pigs significantly enriched GO terms included 
Table 7 Number of IncRNAs in each genomic location group

\begin{tabular}{llll}
\hline & Chicken & Cattle & Pig \\
\hline Sense Intergenic Upstream & 1302 & 843 & 1733 \\
Sense Intergenic Downstream & 1679 & 923 & 1868 \\
Antisense Intergenic Upstream & 2063 & 1747 & 3069 \\
Antisense Intergenic Downstream & 1168 & 790 & 1696 \\
Intergenic, No Gene Within 100 kb & 1208 & 1216 & 3109 \\
Sense Containing Exonic & 227 & 182 & 344 \\
Sense Overlapping Exonic & 48 & 46 & 79 \\
Sense Nested Exonic & 49 & 41 & 109 \\
Sense Containing Intronic & 58 & 30 & 72 \\
Sense Overlapping Intronic & 27 & 25 & 21 \\
Sense Nested Intronic & 166 & 128 & 232 \\
Antisense Containing Exonic & 8 & 12 & 14 \\
Antisense Overlapping Exonic & 465 & 372 & 565 \\
Antisense Nested Exonic & 119 & 75 & 198 \\
Antisense Containing Intronic & 110 & 97 & 205 \\
Antisense Overlapping Intronic & 362 & 418 & 622 \\
Antisense Nested Intronic & 334 & 290 & 493 \\
Total & 9393 & 7235 & 14,429 \\
\hline
\end{tabular}

lung morphogenesis and immune response in pigs and cardiovascular system development in cattle. For muscle, very few terms were significantly enriched in cattle, but muscle tissue development was the most significant. Heart morphogenesis was the most significantly enriched term for muscle in pigs, which only had a total of three significantly enriched GO terms. Chicken had comparatively more significantly enriched terms in muscle, including skeletal muscle development. Finally, lymphocyte or T cell activation were enriched GO terms for spleen in all three species.

\section{Conservation of IncRNAs}

The lncRNAs identified in this study were used to analyze the evolutionary conservation of lncRNAs. In addition to chicken, cattle, and pig, the annotated lncRNAs from human and mouse were included. As the only non-mammal, chicken is the most evolutionarily distant of the species, while cattle and pig are more closely related to each other than to human or mouse (Fig. 4a). Previous studies have shown that lncRNAs are not well conserved at the sequence level [66]. Therefore, positional conservation was analyzed. Using the lncRNA-gene pairs used in the previous analysis (Fig. 2), a lncRNA from one species was considered conserved in another species if the genes paired to each IncRNA were orthologs of each other. There was $\sim 30 \%$ conservation in all species (Fig. 4b, c). A total of 39 ortholog groups were identified containing lncRNAs across the five species, consisting of 64 chicken lncRNAs, 55 cattle lncRNAs, 67 pig lncRNAs, 78 mouse lncRNAs, and 113 human lncRNAs. These lncRNAs are listed with their associated genes in Additional file 20. A GO term analysis of the genes associated with conserved lncRNAs showed that they have functions fundamental to cell biology (Fig. 4d). Chromatin assembly and nucleosome organization appeared in all three farm animal species along with related terms. Multiple sequence alignments performed on each of the groups of lncRNAs (Additional file 21) showed some regions of conservation between the species, although not at the magnitude of what would be expected of orthologous protein-coding genes.

\section{Discussion}

The major goal of this study was to identify tissue-specific lncRNAs, evolutionarily conserved lncRNAs, and their potential regulatory functions across three farm animal genomes using deep RNA sequencing from eight tissues and two biological replicates. A major strength of this study compared to other IncRNA identification studies was the consistency in the methods used to obtain the data across the tissues and species. Because all the data were generated in the same lab by the same personnel and followed the same procedure from the same eight tissues taken from adult males, a comparison of lncRNAs among the three species with limited potential confounding factors such as different developmental stages, tissue types, or sexes was performed. Such a comparison would not have been possible using existing lncRNA annotations from Ensembl or NCBI, or by leveraging lncRNA sets previously identified by other researchers.

\section{Identification of IncRNAs}

The observation that mRNAs contain on average more exons than lncRNAs is consistent with findings from the human ENCODE project [65]. However, no large difference was observed in the length of lncRNAs compared to mRNAs, despite the difference in exon count. This indicates that the exons in lncRNAs were generally larger than in mRNAs. Interestingly, a relatively large percentage of chicken lncRNAs were over 10,000 bp long when compared to both the IncRNAs of cattle and pig, and the mRNAs across all three species. Given the higher depth of RNA-seq achieved compared to the other two species (see Table 1), and the smaller size of the chicken genome (one third that of mammals), this observation may suggest that lncRNA transcripts in close proximity to one another in the genome may be combining during transcript assembly, or un-spliced transcripts may be causing introns to be occasionally sequenced and included in the assembly. In addition, while the majority of both lncRNAs and mRNAs only had a single isoform, this was more pronounced in mRNAs where at least $90 \%$ of genes had a single isoform in all species. This is 


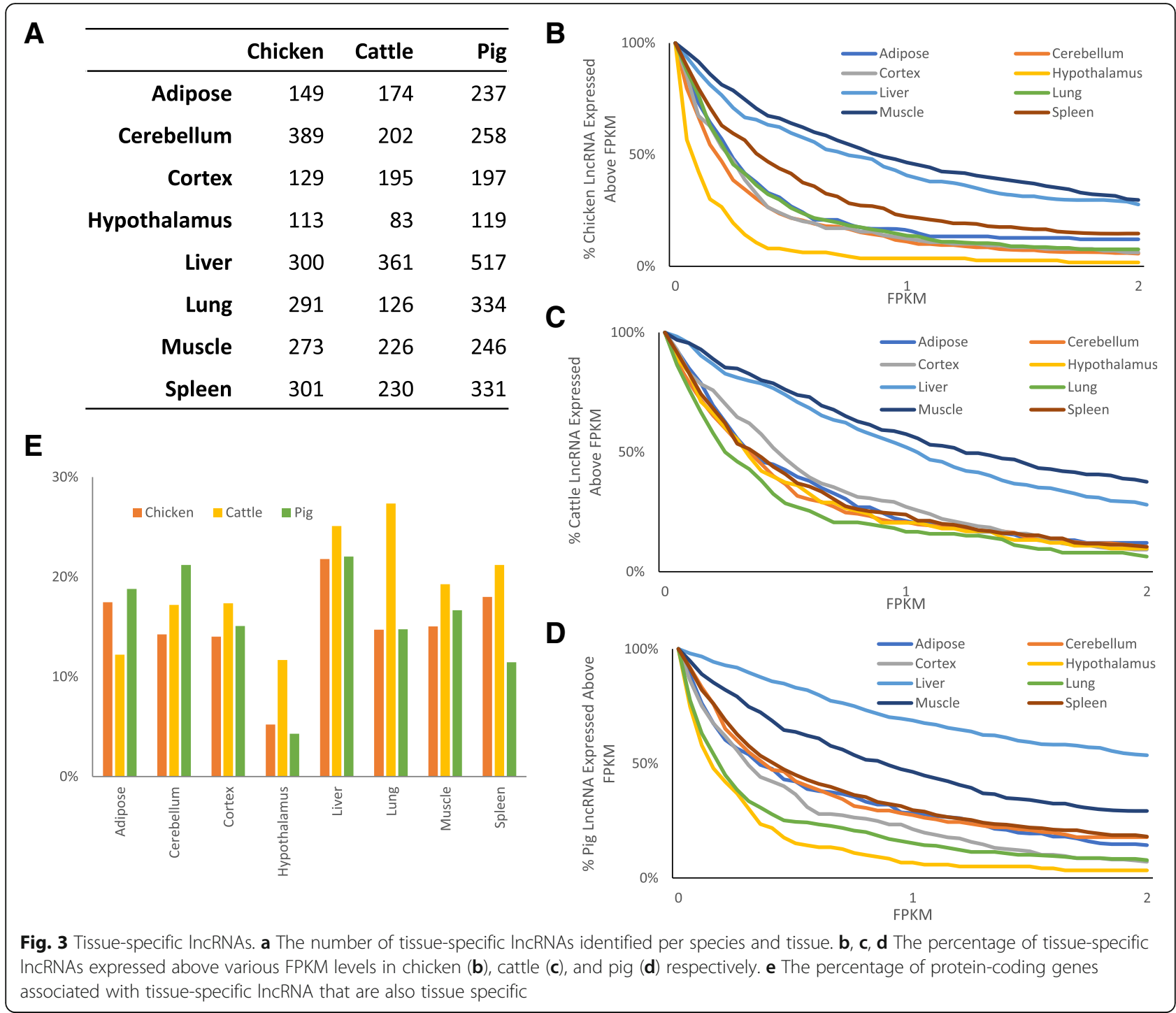

contrary to the results from the ENCODE projects, where IncRNAs had generally fewer isoforms than mRNAs [65]. We speculate that the difference between this study and ENCODE might be an artifact of the transcript assembly and merging process, as many lncRNA isoforms differ only in exon length, not count, and are candidates for merging into a single isoform.

The proportion of IncRNAs categorized into each positional relationship to nearby protein-coding genes was very similar between species, as shown in Fig. 2a-c. However, the percentage of lncRNAs not categorized due to being outside the $50 \mathrm{~kb}$ window of any gene was lowest in chickens, as expected due to their small genome. The chicken genome is roughly one third the size of mammalian genomes, but with a similar number of genes. While the chicken has the lowest rate of excluded lncRNAs, there was still a notable difference between cattle and pig. The quality of the reference genomes and annotations for these species are being continually improved, and so a difference of quality in the current genomes could be causing this disparity.

Across all species, intergenic lncRNAs that were antisense to the nearest protein coding gene showed a prevalence for being upstream of those genes, while IncRNAs that were on the same strand as the nearest protein coding gene were equally upstream and downstream. Because the transcripts are on opposite strands and upstream of each other, they may share a promoter region if they are close enough. This sharing of regulatory regions could allow co-evolution of lncRNA and gene, leading to a higher prevalence of this upstream antisense relationship.

\section{Tissue-specific IncRNAs}

Tissue-specific lncRNAs were identified, resulting in a few hundred per tissue per species (Fig. 3a). The potential 


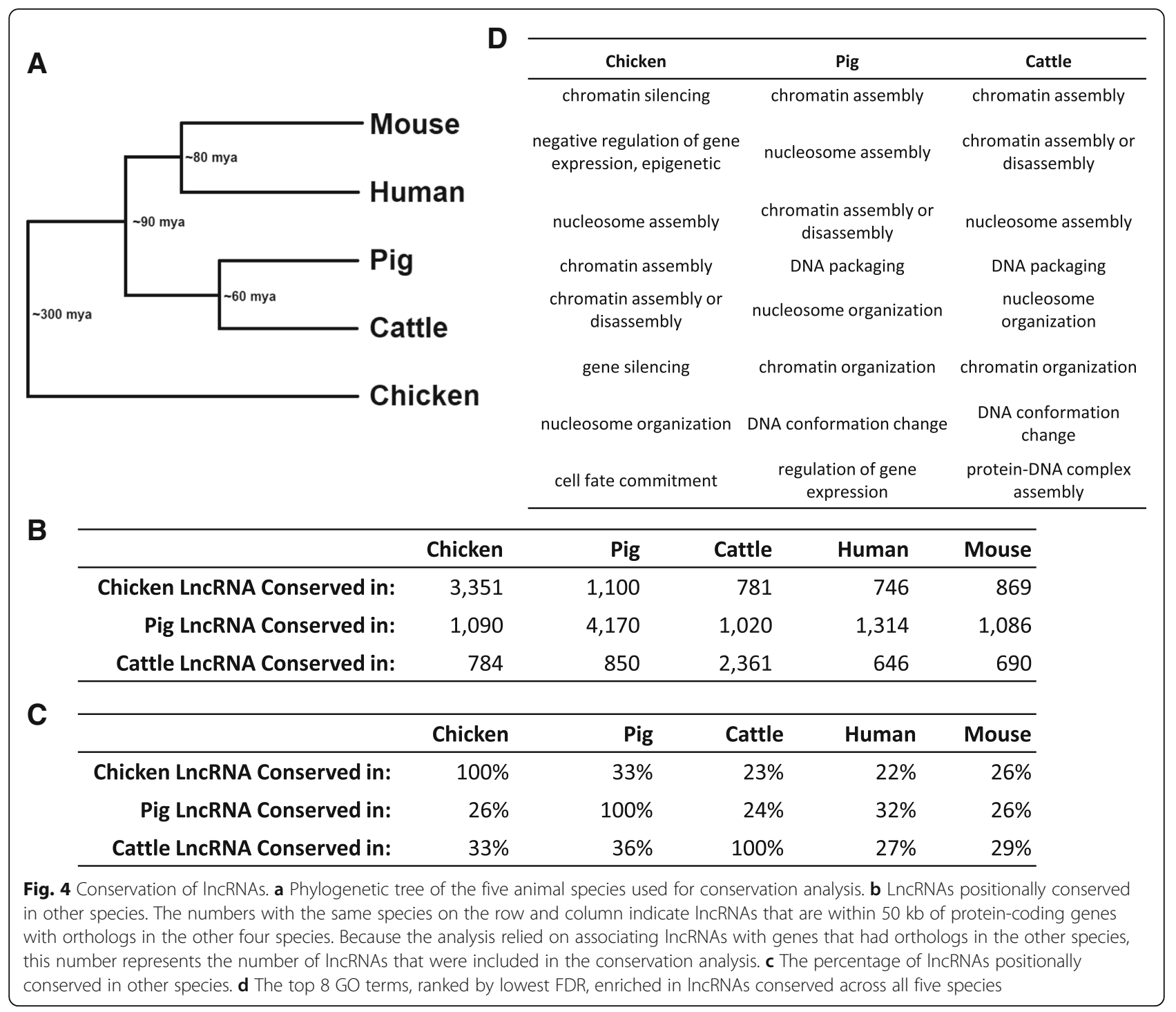

function of these lncRNAs was predicted by examining $\mathrm{GO}$ term enrichment of the nearest protein-coding genes. For many tissues, terms with highly significant enrichment were associated with functions fundamental to those tissues, which has been seen in previous studies of mammalian IncRNAs [67]. Immune system terms, and more specifically lymphocyte activation, were enriched in spleen in all three species, with chicken GO term enrichment even more specific with $\mathrm{T}$ cell activation, which suggests expression of these spleen-specific lncRNAs are important for immune function. GO terms related to circulatory system were prevalent in tissues with a high density of blood vessels. This prevalence was observed across the three species in lung and brain, and in spleen from pigs and chickens. Less than $20 \%$ of genes associated with tissue-specific lncRNAs were themselves tissuespecific in their expression (Fig. 3e). This is not surprising, as studies looking at the regulatory mechanisms of specific
lncRNAs have found both positive and negative regulatory functions, including post-transcriptional regulation [68]. When performing this analysis, an unadjusted $p$-value of 0.01 was used as a significance cutoff, rather than a value adjusted for multiple testing such as false discovery rate (FDR). This choice was made because the assumption that a lncRNA regulates the nearest protein-coding gene is a useful heuristic, but likely produces some false positives which should be considered when interpreting these results. The use of a more relaxed statistical significance cutoff yielded many of the biologically interesting results which would not have been seen using FDR. Unfortunately, few options exist currently to predict the regulatory target of lncRNAs.

\section{Conservation of IncRNAs}

One of the main goals of this study was to identify the conservation of IncRNAs across three evolutionarily 
diverse species. Previous studies have found few conserved sequences across the lncRNAs among different organisms, even among closely related species [66]. Therefore, conservation analysis across species based on synteny was proposed. LncRNAs from the human and mouse NCBI annotations were also included so the conservation across five species could be analyzed. Because the human and mouse data do not have complete consistency in tissue, developmental stage, and sex from the data generated for this study, it was only appropriate to examine the conservation of chicken, cattle, and pig lncRNAs in mouse and human, but not vice versa. While a greater conservation was expected among the four mammalian species than with chicken, this was not reflected in this study's results. This may simply be due to differences in the number of identified lncRNAs, which depends on the reference genome annotation quality. However, it may also suggest that most lncRNAs evolved very quickly and are not well conserved, with a small group of conserved lncRNAs representing evolutionarily ancient sequences. Such a hypothesis is supported by the 39 groups of orthologs that contain a lncRNA from all five species. The GO term analysis of nearby genes yielded biological processes that are common to cells across all eukaryotes, and would therefore be conserved over long evolutionary distances. These lncRNAs have been conserved for at least 300 million years, when the ancestors of birds and mammals diverged, and may be much older.

\section{Conclusions}

This study identified 9393 lncRNA transcripts from 4654 loci in chickens, 7235 lncRNAs from 4325 loci in cattle, and 14,429 lncRNAs from 8772 loci in pigs. About half of these lncRNAs were previously annotated in the NCBI annotations of these species, with the remaining half consisting of approximately 50\% novel transcripts of previously annotated lncRNAs and 50\% lncRNAs identified at loci from which no currently annotated transcript originates.

Synteny-based conservation analysis across five evolutionarily diverse species (farm animals plus mouse and human) revealed a total of 39 distinct groups of lncRNAs. Conserved lncRNAs were associated with coding genes involved in epigenetic regulation and the physical structure of DNA (Fig. 4d).

Tissue-specific lncRNA analysis indicated that a greater proportion of lncRNAs specific to muscle and liver were highly expressed compared to the six other tissues. GO terms of coding genes associated with tissue-specific lncRNAs were enriched for tissue-specific functions. For example, in all three farm animal species, GO terms enriched in spleen were associated with lymphocyte activation and other immune-related GO terms.
This initial analysis revealed many novel insights into potential regulatory roles for lncRNAs with regard to tissue specificity and evolutionary conservation. As a part of ongoing FAANG research, ChIP-seq is being employed using the same tissue samples from this study to profile four histone modifications (H3K4me3, H3K27me3, H3K4me1, and $\mathrm{H} 3 \mathrm{~K} 27 \mathrm{ac}$ ) associated with promoters and enhancers, as well as binding sites for the transcription factor CTCF to identify insulators. This will further our understanding of the epigenetic regulation of protein-coding genes by lncRNAs. Additionally, ISO-seq, for full transcript sequencing, and RAMPAGE [69], for the accurate detection of transcription start sites, efforts are also underway, which will further refine the accuracy of these lncRNA annotations.

\section{Methods}

\section{Genetic resources}

Tissues were collected specifically for this study with all necessary permissions granted, following Protocol for Animal Care and Use \#18464, approved by the Institutional Animal Care and Use Committee (IACUC), University of California, Davis. Animals were euthanized for collection of tissues from adipose, cerebellum, cortex, hypothalamus, liver, lung, skeletal muscle, and spleen and flash frozen in liquid nitrogen, then stored at $-80{ }^{\circ} \mathrm{C}$ until processing. Chickens were euthanized using $\mathrm{CO} 2$ under USDA inspection and samples were collected from two male F1 crosses of Line 6 and Line 7 from the Avian Disease and Oncology Laboratory (ADOL) at 20 weeks of age. Cattle were slaughtered by captive bolt under USDA inspection and samples were collected at University of California, Davis, from two intact male Line 1 Herefords provided by Fort Keogh Livestock and Range Research Lab at 14 months of age. Both individuals shared the same sire. Pigs were humanely slaughtered under USDA inspection and samples were collected from two castrated male littermate Yorkshires at Michigan State University at 6 months of age. The ages for all animals correspond with the sexually mature adult stage for their species.

\section{Library preparation and sequencing}

Total RNA was isolated using Trizol (Invitrogen, Carlsbad, $\mathrm{CA}$ ) according to the manufacturer's protocol. DNase I (Ambion, Austin, TX) digestion was carried out after RNA isolation and the RNA concentration and purity were determined by measuring absorbance at $260 \mathrm{~nm}$ and A260/ A280 ratio using a NanoDrop ND-1000 spectrophotometer (NanoDrop Technologies, Wilmington, DE). RNA samples were stored at $-80{ }^{\circ} \mathrm{C}$ until further use. Total RNA $(1 \mu \mathrm{g})$ was subjected to two rounds of hybridization to oligo (dT) beads (Invitrogen, Carlsbad, CA) to enrich poly-adenylated transcripts. Stranded RNA-seq libraries were prepared using the TruSeq RNA Illumina protocol, and libraries were sequenced on an Illumina HiSeq-3000 
using $100 \mathrm{bp}$ PE to a depth of at least 50 million reads per library, or 100 million reads per tissue (when replicates were combined).

\section{Read mapping and transcript assembly}

Reads were trimmed to remove adapter sequences and low quality bases using the Trim Galore program [70] with default parameters. TopHat 2 was used with default parameters to align reads to their respective genomes [71]. Genome assemblies and annotations were obtained from NCBI, using Galgal5 (annotation release 103) for chicken, Sscrofa10.2 (annotation release 105) for pig, and UMD3.1.1 (annotation release 105) for cattle. No annotation was used during the alignment step to avoid biasing the alignments towards previously annotated splice junctions. Alignments were then filtered with the samtools view '-q 15' parameter to remove those with a MAPQ alignment score of less than 15, which removes low quality alignments and multi-mapped reads. Cufflinks was run on each library individually with the library-type' parameter set to 'fr-firststrand' and with a modified NCBI annotation, containing only the protein-coding genes, provided using the '-g' parameter. Transcriptomes were then combined using Cuffmerge with the NCBI annotation provided using the '-g' parameter to generate a set of transcripts whose expression levels could be measured across tissues [72]. Final expression levels were generated using Cuffnorm with the combined GTF file output by Cuffmerge and with the '-library-norm-method' parameter set to 'geometric' and 'library-type' parameter set to 'fr-firststrand'.

\section{Identification of LncRNAs}

Genome annotations from NCBI were used to match assembled transcripts with known genes. As mentioned in the previous section, annotated non-coding transcripts were removed from the annotations by filtering elements that did not have 'gene_biotype = protein_coding' so that only protein-coding genes were used to filter assembled transcripts in order to create a completely de novo set of lncRNAs. Any transcript with a Cufflinks class code of "=", indicating a transcript matching an annotated gene, was removed from the combined set of transcripts. To reduce false positives, mono-exonic transcripts were also omitted, as they are likely to be transcriptional noise. The remaining sequences were then aligned to the Swiss-Prot database [62] to identify homology with known proteins, as well as the Pfam-A database [61] to locate protein domains. Protein sequences were downloaded from their respective websites and NCBI-BLAST [73] was used with the blastx algorithm with default parameters to align translated RNA to the protein databases. Any transcript with a hit in either of these databases with an e-value less than 0.001 was removed, leaving the final set of long non-coding RNAs (lncRNAs). Coding potential scores were calculated for every lncRNA using FEELnc [64] with default parameters. For positive training data, mRNA sequences from the NCBI annotation with "gene_biotype = protein_coding" were used. The negative training data used were the lncRNA sequences from the NONCODEv5 database [63] for the species being analyzed. These scores are shown in Additional files 22, 23 and 24. Note that the coding potential scores were not used in the prediction of the IncRNA, but were calculated and provided as a confidence metric. Overlap of the predicted lncRNA with the NONCODEv5 database was determined using NCBI-BLAST with the blastn command. An evalue cutoff of 1e-5, percentage identity (pident in tabular output parameter) greater than $50 \%$, and query coverage (qcovs in tabular output parameter) greater than $50 \%$ was used. All other parameters were default. A few IncRNA were tested with PCR to validate they were not genomic DNA contamination. This is shown in Additional file 25.

\section{Correlation of expression of IncRNA and nearby protein- coding genes}

The correlation between IncRNA and nearby proteincoding genes was calculated using Spearman correlation, which ranks both sets of expression values and calculates the Pearson correlation based on ranks rather than raw expression values. No cutoff value was used and all pairs of lncRNA and protein-coding genes were included in the calculation.

\section{Tissue-specific LncRNAs identification}

Tissue-specific lncRNAs were identified using the tissue specificity index (TSI) [74]. TSI is defined as:

$$
\tau=\frac{\sum_{i=1}^{N}\left(1-x_{i}\right)}{N-1}
$$

where $N$ is the number of tissues and $x_{i}$ is the expression of the lncRNA $x$ in tissue $i$ normalized by the maximum expression value. Transcripts with a TSI of greater than 0.9 in both replicates were considered tissue specific. This threshold is recommended in Yanai, et al. [74]. As previously described, Cuffnorm was used to measure expression values, using the "-library-norm-method" parameter set to "geometric". This uses a normalization method similar to DESeq rather than the default method of calculating FPKM, which is now considered obsolete in favor of TPM. Enriched GO terms were determined using the DAVID Bioinformatics Resource version 6.8 $[75,76]$ with the default parameters. A $p$-value cutoff of 0.01 was used to consider significant enrichment. The gene list input into DAVID contained every gene from the lncRNA-gene pairs for every lncRNA specific to the 
tissue. The background was the default set used by DAVID, which is the entire set of genes for the species.

\section{Conservation of LncRNAs}

NCBI BLAST + 2.2.29 [73] was used to align lncRNA sequences to each other across species. Alignment was done using default parameters as well as using the relaxed parameters "-word_size 7 -reward 1 -penalty -2". To identify orthologous pairs, a reciprocal method was used, requiring that the best scoring hit (measured by e-value) when aligning species A to species B matched the best scoring hit when aligning the opposite direction, species B to species A. Only alignments with an e-value under the threshold of 10e-5 were used.

OrthoFinder (0.2.8) [77] was used with default arguments to identify groups of orthologs using the NCBI RefSeq proteins for chicken, cattle, pig, human, and mouse. The proteins were then mapped to genes, and only the groups containing at least one gene from all five species (12,390 groups) were kept for further downstream analysis. The classifier function of FEELnc [64] was used to associate lncRNAs with genes within 50,000 bp upstream or downstream, a distance cut-off used in previous studies [78]. LncRNAs from different species that are associated with genes in the same ortholog group are considered putative orthologs. Enriched GO terms were determined using DAVID as described in the previous subsection. To generate multiple sequence alignments of the lncRNAs in the conserved groups, ClustalW (2.1) was used with default parameters [79].

\section{Additional files}

Additional file 1: Sequences of Chicken LncRNAs. A fasta file containing all the IncRNA sequences from chickens. (FA $34950 \mathrm{~kb}$ )

Additional file 2: Sequences of Cattle LncRNAs. A fasta file containing all the IncRNA sequences from cattle. (FA $14461 \mathrm{~kb}$ )

Additional file 3: Sequences of Pig LncRNAs. A fasta file containing all the IncRNA sequences from pigs. (FA $21227 \mathrm{~kb}$ )

Additional file 4: Exon Locations of Chicken LncRNAs. The genomic locations of the exons of all chicken IncRNAs. (GTF $7220 \mathrm{~kb}$ )

Additional file 5: Exon Locations of Cattle LncRNAs. The genomic locations of the exons of all cattle IncRNAs. (GTF $4359 \mathrm{~kb}$ )

Additional file 6: Exon Locations of Pig LncRNAs. The genomic locations of the exons of all pig IncRNAs. (GTF $9045 \mathrm{~kb}$ )

Additional file 7: Mapping to NONCODE. The NONCODE IDs for each IncRNA found in the NONCODE database, as described in the Methods section. For IncRNA that had multiple matches in the NONCODE database, the NONCODE ID for the match with the highest bit score is used. (XLSX $151 \mathrm{~kb}$ )

Additional file 8: LncRNA Classes from Chickens. (TSV 3281 kb)

Additional file 9: LncRNA Classes from Cattle. The output from the FEELnc Classifier program, which finds nearby protein-coding genes for each IncRNA and classifies their positional relationship. See Additional file 8 for column descriptions. (TSV $1794 \mathrm{~kb}$ )

Additional file 10: LncRNA Classes from Pigs. The output from the FEELnc Classifier program, which finds nearby protein-coding genes for each IncRNA and classifies their positional relationship. See Additional file 8 for column descriptions. (TSV $3182 \mathrm{~kb}$ )

Additional file 11: Expression of Chicken LncRNAs. FPKM values for all IncRNAs from each RNA-seq library (2 libraries per tissue) in chickens. (TSV $12559 \mathrm{~kb}$ )

Additional file 12: Expression of Cattle LncRNAs. FPKM values for all IncRNAs from each RNA-seq library (2 libraries per tissue) in cattle. (TSV $15586 \mathrm{~kb}$ )

Additional file 13: Expression of Pig LncRNAs. FPKM values for all IncRNAs from each RNA-seq library (2 libraries per tissue) in pigs. (TSV 14242 kb)

Additional file 14: Tissue-specific Indices of Chicken LncRNAs. The calculated tissue-specific indices (TSI) for each IncRNA in each tissue. The "TSI A" and "TSI B" columns are the TSI calculated for each biological replicate. The "TSI Both" column is the F1 score of the TSI from both replicates. F1 is calculated as $\left(2{ }^{*} A * B\right) /(A+B)$ where A is TSI A and B is TSI. The "Name" and "Location" columns give the ID and genomic location of the IncRNA, and the following columns are the FPKM values from each library. (XLSX $1551 \mathrm{~kb}$ )

Additional file 15: Tissue-specific Indices of Cattle LncRNAs. The calculated tissue-specific indices (TSI) for each IncRNA in each tissue. The "TSI A" and "TSI B" columns are the TSI calculated for each biological replicate. The "TSI Both" column is the F1 score of the TSI from both replicates. F1 is calculated as $(2 * A * B) /(A+B)$ where A is TSI A and B is TSI. The "Name" and "Location" columns give the ID and genomic location of the IncRNA, and the following columns are the FPKM values from each library. (XLSX $1122 \mathrm{~kb}$ )

Additional file 16: Tissue-specific Indices of Pig LncRNAs. The calculated tissue-specific indices (TSI) for each IncRNA in each tissue. The "TSI A" and "TSI B" columns are the TSI calculated for each biological replicate. The "TSI Both" column is the F1 score of the TSI from both replicates. F1 is calculated as $\left(2{ }^{*} A{ }^{*} B\right) /(A+B)$ where $A$ is TSI A and B is TSI. The "Name" and "Location" columns give the ID and genomic location of the IncRNA, and the following columns are the FPKM values from each library. (XLSX $1754 \mathrm{~kb}$ )

Additional file 17: GO terms associate with tissue-specific IncRNAs in chickens. (XLSX $149 \mathrm{~kb}$ )

Additional file 18: GO terms associate with tissue-specific IncRNAs in cattle. This file contains tables from the DAVID analysis tool for each of the eight tissues, showing GO terms enriched by genes associated with tissue-specific IncRNAs in cattle. GO terms outside a significance cutoff of $p$-value $<0.01$ are shaded in red. See Additional file 17 for column descriptions. (XLSX $171 \mathrm{~kb}$ )

Additional file 19: GO terms associate with tissue-specific IncRNAs in pigs. This file contains tables from the DAVID analysis tool for each of the eight tissues, showing GO terms enriched by genes associated with tissue-specific IncRNAs in pigs. GO terms outside a significance cutoff of $p$-value $<0.01$ are shaded in red. See Additional file 17 for column descriptions. (XLSX $144 \mathrm{~kb})$

Additional file 20: Conserved LnCRNAs. The 39 groups of orthologous genes across all five species are listed with the associated IncRNAs. Human and mouse IncRNA IDs are NCBI transcript IDs. (XLSX 21 kb)

Additional file 21: Multiple Sequence Alignments for Conserved LncRNAs. A multiple sequence alignment file generated by ClustalW is included for each of the 39 groups of IncRNAs associated with orthologous genes across all five species. (ZIP $445 \mathrm{~kb}$ )

Additional file 22: FEELnc Coding Potential Scores for Chicken LncRNAs. The coding potential scores calculated by FEELnc. The "coding_potential" column is the coding potential score, with 0 being the least likely to be coding and 1 being most likely. The "label" column is 0 or 1 to indicate if the score lies above or below the cutoff determined by FEELnc using cross validation. 0 indicates a predicted IncRNA while 1 is a predicted coding transcript. (XLSX $217 \mathrm{~kb}$ )

Additional file 23: FEELnc Coding Potential Scores for Cattle LnCRNAs. The coding potential scores calculated by FEELnc. The "coding_potential" column is the coding potential score, with 0 being the least likely to be coding and 1 being most likely. The "label" column is 0 or 1 to indicate if the score lies above or below the cutoff determined by FEELnc using cross validation. 0 indicates a predicted IncRNA while 1 is a predicted coding transcript. (XLSX $173 \mathrm{~kb}$ ) 
Additional file 24: FEELnc Coding Potential Scores for Pig LncRNAs. The coding potential scores calculated by FEELnc. The "coding_potential" column is the coding potential score, with 0 being the least likely to be coding and 1 being most likely. The "label" column is 0 or 1 to indicate if the score lies above or below the cutoff determined by FEELnc using cross validation. 0 indicates a predicted IncRNA while 1 is a predicted coding transcript. (XLSX $323 \mathrm{~kb}$ )

Additional file 25: RT-PCR Gel Images for Validation. Gel images from a few RT-PCRs to verify a few of the predicted IncRNAs. (PPTX $203 \mathrm{~kb}$ )

\section{Funding}

This work was supported by United States Department of Agriculture, National Institute of Food and Agriculture (Competitive Grant Project no. 2015-67015-22940 to HZ). Further support was provided by United States Department of Agriculture, National Institute of Food and Agriculture, National Animal Genome Research Program, U.S. Poultry, Cattle, and Swine Genome Coordination Fund, National Pork Board, and Aviagen, and United States Department of Agriculture, National Institute of Food and Agriculture, Multistate Research Project NRSP8 and NC1170 (HZ) and the California Agricultural Experimental Station (MED, HZ).

\section{Availability of data and materials}

The raw sequencing data and BioSample accessions used for this study are available from the European Nucleotide Archive under project ID PRJEB14330 (https://www.ebi.ac.uk/ena/data/view/PRJEB14330).

\section{Authors' contributions}

CK performed all bioinformatics analysis and wrote the manuscript. YW performed RNA extractions the 48 tissues used for the study. JC prepared and submitted all libraries for sequencing. IK, MD, HC, JM, AvE, CE, PR, and $\mathrm{HZ}$ contributed significantly to the experimental design. PR and $\mathrm{HZ}$ supervised the whole study. All authors provided feedback while the manuscript was being drafted and approved the final version.

\section{Ethics approval and consent to participate}

Protocol for Animal Care and Use \#18464, "Genome wide identification and annotation of functional regulatory regions in livestock species", was approved by the Institutional Animal Care and Use Committee (IACUC), University of California, Davis.

\section{Consent for publication}

Not applicable.

\section{Competing interests}

Author Hans Cheng is a member of the editorial board (Associate Editor) of this journal.

\section{Publisher's Note}

Springer Nature remains neutral with regard to jurisdictional claims in published maps and institutional affiliations.

\section{Author details}

'Department of Animal Science, University of California, Davis, Davis, CA, USA. ${ }^{2}$ Genome Center, University of California, Davis, Davis, CA, USA.

${ }^{3}$ USDA-ARS, Avian Disease and Oncology Laboratory, East Lansing, MI, USA. ${ }^{4}$ Department of Animal Science, Michigan State University, East Lansing, MI, USA.

\section{Received: 19 December 2017 Accepted: 27 August 2018}

\section{Published online: 18 September 2018}

\section{References}

1. Carninci $P$, et al. The transcriptional landscape of the mammalian genome. Science. 2005;309(5740):1559-63.

2. Kapranov P, et al. Examples of the complex architecture of the human transcriptome revealed by RACE and high-density tiling arrays. Genome Res. 2005;15(7):987-97.

3. Kapranov P, Willingham AT, Gingeras TR. Genome-wide transcription and the implications for genomic organization. Nat Rev Genet. 2007;8(6):413-23.
4. Mouse Genome Sequencing, $C$, et al. Initial sequencing and comparative analysis of the mouse genome. Nature. 2002;420(6915):520-62.

5. Gibbs RA, et al. Genome sequence of the Brown Norway rat yields insights into mammalian evolution. Nature. 2004:428(6982):493-521.

6. Venter JC, et al. The sequence of the human genome. Science. 2001; 291(5507):1304-51.

7. Lander ES, et al. Initial sequencing and analysis of the human genome. Nature. 2001;409(6822):860-921.

8. Ruiz-Orera J, et al. Translation of neutrally evolving peptides provides a basis for de novo gene evolution. Nat Ecol Evol. 2018;2(5):890-6.

9. Wilson BA, Masel J. Putatively noncoding transcripts show extensive association with ribosomes. Genome Biol Evol. 2011;3:1245-52.

10. Clemson CM, et al. XIST RNA paints the inactive $X$ chromosome at interphase: evidence for a novel RNA involved in nuclear/chromosome structure. J Cell Biol. 1996;132(3):259-75.

11. Gupta RA, et al. Long non-coding RNA HOTAIR reprograms chromatin state to promote cancer metastasis. Nature. 2010;464(7291):1071-6.

12. Zhang B, et al. The IncRNA Malat1 is dispensable for mouse development but its transcription plays a cis-regulatory role in the adult. Cell Rep. 2012; 2(1):111-23

13. Khalil AM, et al. Many human large intergenic noncoding RNAs associate with chromatin-modifying complexes and affect gene expression. Proc Natl Acad Sci U S A. 2009;106(28):11667-72.

14. Jia $\mathrm{H}$, et al. Genome-wide computational identification and manual annotation of human long noncoding RNA genes. RNA. 2010;16(8):1478-87.

15. Ørom UA, et al. Long noncoding RNAs with enhancer-like function in human cells. Cell. 2010;143(1):46-58.

16. Cabili MN, et al. Integrative annotation of human large intergenic noncoding RNAs reveals global properties and specific subclasses. Genes Dev. 2011;25(18):1915-27.

17. Derrien T, et al. The GENCODE v7 catalog of human long noncoding RNAs: analysis of their gene structure, evolution, and expression. Genome Res. 2012;22(9):1775-89.

18. Sigova AA, et al. Divergent transcription of long noncoding RNA/mRNA gene pairs in embryonic stem cells. Proc Natl Acad Sci U S A. 2013;110(8): 2876-81.

19. Ravasi T, et al. Experimental validation of the regulated expression of large numbers of non-coding RNAs from the mouse genome. Genome Res. 2006; 16(1):11-9.

20. Ponjavic J, Ponting CP. The long and the short of RNA maps. Bioessays. 2007:29(11):1077-80

21. Guttman M, et al. Chromatin signature reveals over a thousand highly conserved large non-coding RNAs in mammals. Nature. 2009;458(7235): 223-7.

22. Guttman M, et al. Ab initio reconstruction of cell type-specific transcriptomes in mouse reveals the conserved multi-exonic structure of lincRNAs. Nat Biotechnol. 2010;28(5):503-10.

23. Ulitsky I, et al. Conserved function of lincRNAs in vertebrate embryonic development despite rapid sequence evolution. Cell. 2011;147(7):1537-50

24. Pauli A, et al. Systematic identification of long noncoding RNAs expressed during zebrafish embryogenesis. Genome Res. 2012;22(3):577-91.

25. Tan $M H$, et al. RNA sequencing reveals a diverse and dynamic repertoire of the Xenopus tropicalis transcriptome over development. Genome Res. 2013; 23(1):201-16.

26. Tupy $\mathrm{LL}$, et al. Identification of putative noncoding polyadenylated transcripts in Drosophila melanogaster. Proc Natl Acad Sci U S A. 2005; 102(15):5495-500.

27. Young RS, et al. Identification and properties of 1,119 candidate lincRNA loci in the Drosophila melanogaster genome. Genome Biol Evol. 2012;4(4): $427-42$.

28. Nam J-W, Bartel DP. Long noncoding RNAs in C. elegans. Genome Res. 2012;22(12):2529-40

29. Liu J, et al. Genome-wide analysis uncovers regulation of long intergenic noncoding RNAs in Arabidopsis. Plant Cell. 2012;24(11):4333-45.

30. Boerner S, McGinnis KM. Computational identification and functional predictions of long noncoding RNA in Zea mays. PLoS One. 2012;7(8):e43047.

31. Broadbent KM, et al. A global transcriptional analysis of plasmodium falciparum malaria reveals a novel family of telomere-associated IncRNAs. Genome Biol. 2011;12(6):R56.

32. Li T, et al. Identification of long non-protein coding RNAs in chicken skeletal muscle using next generation sequencing. Genomics. 2012;99(5):292-8. 
33. Li A, et al. Genome-scale identification of miRNA-mRNA and miRNAIncRNA interactions in domestic animals. Anim Genet. 2015;46(6):716-9.

34. Chodroff RA, et al. Long noncoding RNA genes: conservation of sequence and brain expression among diverse amniotes. Genome Biol. 2010;11(7):R72.

35. Necsulea A, et al. The evolution of IncRNA repertoires and expression patterns in tetrapods. Nature. 2014:505(7485):635-40.

36. Muret $\mathrm{K}$, et al. Long noncoding RNA repertoire in chicken liver and adipose tissue. Genet Sel Evol. 2017:49:6.

37. Zhang $\mathrm{T}$, et al. Genome-wide analysis of IncRNA and mRNA expression during differentiation of abdominal preadipocytes in the chicken. G3. 2017; 7(3):953-66.

38. Huang W, Long N, Khatib H. Genome-wide identification and initial characterization of bovine long non-coding RNAs from EST data. Anim Genet. 2012;43(6):674-82.

39. Weikard R, Hadlich F, Kuehn C. Identification of novel transcripts and noncoding RNAs in bovine skin by deep next generation sequencing. BMC Genomics. 2013;14:789.

40. Billerey $\mathrm{C}$, et al. Identification of large intergenic non-coding RNAs in bovine muscle using next-generation transcriptomic sequencing. BMC Genomics. 2014;15:499

41. Koufariotis LT, et al. A catalogue of novel bovine long noncoding RNA across 18 tissues. PLoS One. 2015;10(10):e0141225.

42. Tong $C_{\text {, et }}$ al. Identification and characterization of long intergenic noncoding RNAs in bovine mammary glands. BMC Genomics. 2017;18(1):468.

43. Liu XF, et al. An atlas and analysis of bovine skeletal muscle long noncoding RNAs. Anim Genet. 2017;48(3):278-86.

44. Ren $\mathrm{H}$, et al. Genomic structure, chromosomal localization and expression profile of a porcine long non-coding RNA isolated from long SAGE libraries. Anim Genet. 2009;40(4):499-508.

45. Xiao B, et al. Identification, bioinformatic analysis and expression profiling of candidate mRNA-like non-coding RNAs in Sus scrofa. J Genet Genomics. 2009:36(12):695-702.

46. Esteve-Codina A, et al. Exploring the gonad transcriptome of two extreme male pigs with RNA-seq. BMC Genomics. 2011;12(1):552.

47. $\mathrm{Yu}$ L, et al. Comparative analyses of long non-coding RNA in lean and obese pig. Oncotarget. 2017:8(25):41440-50.

48. Zhao $P$, et al. Profiling long noncoding RNA of multi-tissue transcriptome enhances porcine noncoding genome annotation. Epigenomics. 2017;10(3): 301-20.

49. Miao $X$, et al. Ovarian transcriptomic study reveals the differential regulation of miRNAs and IncRNAs related to fecundity in different sheep. Sci Rep. 2016;6:35299.

50. Bakhtiarizadeh MR, et al. In silico prediction of long intergenic non-coding RNAs in sheep. Genome. 2016;59(4):263-75.

51. Ren C, et al. Genome-wide analysis reveals extensive changes in LncRNAs during skeletal muscle development in Hu sheep. Genes. 2017:8(8):191.

52. Zhang $Y$, et al. Long noncoding RNA expression profile changes associated with dietary energy in the sheep testis during sexual maturation. Sci Rep. 2017:7(1):5180

53. Ren $H$, et al. Genome-wide analysis of long non-coding RNAs at early stage of skin pigmentation in goats (Capra hircus). BMC Genomics. 2016;17:67.

54. Zhan S, et al. Genome-wide identification and characterization of long noncoding RNAs in developmental skeletal muscle of fetal goat. BMC Genomics. 2016;17:666.

55. Ling $Y$, et al. Identification and analysis of differentially expressed long noncoding RNAs between multiparous and uniparous goat (Capra hircus) ovaries. PLoS One. 2017;12(9):e0183163.

56. Gao X, et al. Screening and evaluating of long noncoding RNAs in the puberty of goats. BMC Genomics. 2017;18:164.

57. Scott EY, et al. Identification of long non-coding RNA in the horse transcriptome. BMC Genomics. 2017;18(1):511.

58. Weikard R, Demasius W, Kuehn C. Mining long noncoding RNA in livestock. Anim Genet. 2016:48(1):3-18

59. Andersson $\mathrm{L}$, et al. Coordinated international action to accelerate genometo-phenome with FAANG, the functional annotation of animal genomes project. Genome Biol. 2015;16:57.

60. Merkin J, et al. Evolutionary dynamics of gene and Isoform regulation in mammalian tissues. Science. 2012;338(6114):1593.

61. Finn RD, et al. Pfam: the protein families database. Nucleic Acids Res. 2014 42(Database issue):D222-30.
62. Boeckmann B, et al. The SWISS-PROT protein knowledgebase and its supplement TrEMBL in 2003. Nucleic Acids Res. 2003:31(1):365-70.

63. Fang S, et al. NONCODEV5: a comprehensive annotation database for long non-coding RNAs. Nucleic Acids Res. 2018;46(Database issue):D308-14.

64. Wucher $V$, et al. FEELnc: a tool for long non-coding RNA annotation and its application to the dog transcriptome. Nucleic Acids Res. 2017; 45(8):e57.

65. Harrow J, et al. GENCODE: the reference human genome annotation for the ENCODE project. Genome Res. 2012;22(9):1760-74.

66. Hyashizaki Y. Mouse transcriptome: neutral evolution of 'non-coding' complementary DNAs (reply). Nature. 2004;431(7010):1.

67. Washietl S, Kellis M, Garber M. Evolutionary dynamics and tissue specificity of human long noncoding RNAs in six mammals. Genome Res. 2014;24(4): 616-28.

68. Ponting CP, Oliver PL, Reik W. Evolution and functions of long noncoding RNAs. Cell. 2009;136(4):629-41.

69. Batut $P$, Gingeras TR. RAMPAGE: promoter activity profiling by paired-end sequencing of 5'-complete cDNAs. In: Current protocols in molecular biology: Wiley; 2001

70. Krueger F. Trim galore!: a wrapper tool around Cutadapt and FastQC to consistently apply quality and adapter trimming to FastQ files. 2015

71. Trapnell C, Pachter L, Salzberg SL. TopHat: discovering splice junctions with RNA-Seq. Bioinformatics. 2009;25(9):1105-11

72. Trapnell C, et al. Differential gene and transcript expression analysis of RNAseq experiments with TopHat and cufflinks. Nat Protoc. 2012;7(3):562-78.

73. Camacho C, et al. BLAST+: architecture and applications. BMC Bioinf. 2009. 10:421.

74. Yanai I, et al. Genome-wide midrange transcription profiles reveal expression level relationships in human tissue specification. Bioinformatics. 2005;21(5):650-9.

75. Huang DW, Sherman BT, Lempicki RA. Systematic and integrative analysis of large gene lists using DAVID bioinformatics resources. Nat Protoc. 2008:4:44

76. Huang DW, Sherman BT, Lempicki RA. Bioinformatics enrichment tools: paths toward the comprehensive functional analysis of large gene lists. Nucleic Acids Res. 2009;37(1):1-13.

77. Emms DM, Kelly S. OrthoFinder: solving fundamental biases in whole genome comparisons dramatically improves orthogroup inference accuracy. Genome Biol. 2015;16(1):157.

78. Ulitsky I, Bartel DP. lincRNAs: genomics, evolution, and mechanisms. Cell. 2013:154(1):26-46

79. Larkin MA, et al. Clustal W and Clustal X version 2.0. Bioinformatics. 2007; 23(21):2947-8
Ready to submit your research? Choose BMC and benefit from:

- fast, convenient online submission

- thorough peer review by experienced researchers in your field

- rapid publication on acceptance

- support for research data, including large and complex data types

- gold Open Access which fosters wider collaboration and increased citations

- maximum visibility for your research: over $100 \mathrm{M}$ website views per year

At BMC, research is always in progress.

Learn more biomedcentral.com/submissions 